\title{
The young, tight, and low-mass binary TWA22AB: a new calibrator for evolutionary models?^
}

\section{Orbit, spectral types, and temperature}

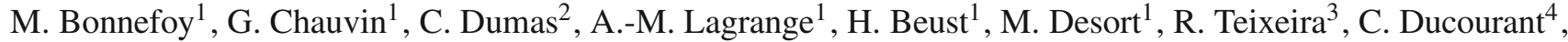 \\ J.-L. Beuzit ${ }^{1}$, and I. Song ${ }^{5}$ \\ 1 Laboratoire d'Astrophysique de Grenoble, BP 53, 38041 Grenoble Cedex 9, France \\ e-mail: mickael . bonnefoy@obs.ujf-grenoble.fr \\ 2 ESO, Alonso de Cordova 3107, Vitacura, Casilla 19001, Santiago 19, Chile \\ 3 Instituto de Astronomia, Geofísica e Ciências Atmosféricas, Universidade de São Paulo, Rua do Matão, 1226 - Cidade Universitária, \\ 05508-900 São Paulo - SP, Brazil \\ 4 Observatoire Aquitain des Sciences de l'Univers, CNRS-UMR 5804, BP 89, 33270 Floirac, France \\ 5 Department of Physics and Astronomy, University of Georgia, Athens, GA 30602, USA
}

Received 5 September 2008 / Accepted 17 May 2009

\begin{abstract}
Context. Tight binaries discovered in young, nearby associations are ideal targets for providing dynamical mass measurements to test the physics of evolutionary models at young ages and very low masses.

Aims. We report the binarity of TWA22 for the first time. We aim at monitoring the orbit of this young and tight system to determine its total dynamical mass using an accurate distance determination. We also intend to characterize the physical properties (luminosity, effective temperature, and surface gravity) of each component based on near-infrared photometric and spectroscopic observations. Methods. We used the adaptive-optics assisted imager NACO to resolve the components, to monitor the complete orbit and to obtain the relative near-infrared photometry of TWA22 AB. The adaptive-optics assisted integral field spectrometer SINFONI was also used to obtain medium-resolution $\left(R_{\lambda}=1500-2000\right)$ spectra in $J H K$ bands. Comparison with empirical and synthetic librairies were necessary for deriving the spectral type, the effective temperature, and the surface gravity for each component of the system. Results. Based on an accurate trigonometric distance $(17.5 \pm 0.2 \mathrm{pc})$ determination, we infer a total dynamical mass of $220 \pm 21 M_{\text {Jum }}$ for the system. From the complete set of spectra, we find an effective temperature $T_{\text {eff }}=2900_{-200}^{+200} \mathrm{~K}$ for TWA22 A and $T_{\text {eff }}=2900_{-100}^{+200} \mathrm{~K}$ for TWA22 B and surface gravities between 4.0 and 5.5 dex. From our photometry and an M6 \pm 1 spectral type for both components, we find luminosities of $\log \left(L / L_{\odot}\right)=-2.11 \pm 0.13$ dex and $\log \left(L / L_{\odot}\right)=-2.30 \pm 0.16$ dex for TWA22 A and B, respectively. By comparing these parameters with evolutionary models, we question the age and the multiplicity of this system. We also discuss a possible underestimation of the mass predicted by evolutionary models for young stars close to the substellar boundary.
\end{abstract}

Key words. stars: fundamental parameters - stars: low-mass, brown dwarfs - binaries: close - stars: formation instrumentation: adaptive optics - instrumentation: spectrographs

\section{Introduction}

Mass and age are fundamental parameters of stars and brown dwarfs that determine their luminosity, effective temperature, atmospheric composition, and surface gravity as commonly derived through photometric and spectroscopic observations. Evolutionary models are currently widely used in the community to infer masses of stars and brown dwarfs, but they rely on equations of states and atmospheric models that are not calibrated at young ages and at very low masses. However, direct mass measurements can be obtained by the means of different observing techniques such as the astrometric follow-up of double-lined spectroscopic tight binaries, the measurment of the Keplerian motion of circumstellar disks or the joint use of light curve and radial velocity on eclipsing binaries. In recent

\footnotetext{
* Based on service-mode observations (072.C-0644, 073.C-0469, 075.C-0521, 076.C-0554, 078.C-0510, 080.C-0581) collected at the European Organisation for Astronomical Research in the Southern Hemisphere, Chile.
}

years, direct mass measurements for 23 pre-main sequence stars with masses ranging from 0.5 to $2 M_{\odot}$ showed discrepancies with predictions by up to a factor of 2 in mass and 10 in ages (Mathieu et al. 2007). Such measurements are rares for lower masses $\left(\leq 0.5 M_{\odot}\right)$ systems. Hillenbrand \& White (2004) show that the models tend to understimate the mass of the companion UZ Tau Eb $\left(M=0.294 \pm 0.027 M_{\odot}\right.$, age $\sim 5 \mathrm{Myr}$, see Prato et al. 2002). Close et al. (2005) derived similar conclusions, but the age and the luminosity of the companion $\mathrm{AB}$ Dor $\mathrm{C}$ ( $M=0.090 \pm 0.005 M_{\odot}$, age $\sim 75 \mathrm{Myr}$ ) is still under debate (Boccaletti et al. 2008). And recently, the surprising discovery of the unpredicted temperature reversal (Stassun et al. 2007) between $2 \mathrm{M} 035 \mathrm{~A}\left(M=0.0541 \pm 0.0046 M_{\odot}\right.$, age $\left.\sim 1 \mathrm{Myr}\right)$ and its companion $\left(M=0.0340 \pm 0.0027 M_{\odot}\right.$, age $\left.\sim 1 \mathrm{Myr}\right)$ has proven the necessity for finding more calibrators. The challenge is to unambiguously determine their physical properties (mass, $L, T_{\text {eff }}$, $g$, and age) and to explore the parameter space covered by evolutionary models as much as possible. The influence of other 


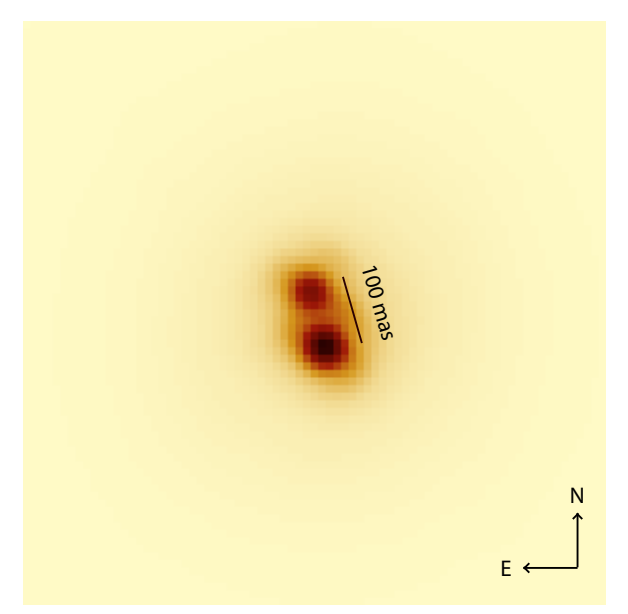

Fig. 1. VLT/NACO image of TWA22 AB obtained in $H$-band with the S13 camera on 2007 December 26. North is up and east is left. The field of view is $1^{\prime \prime} \times 1^{\prime \prime}$.

parameters such as metallicity also needs investigation (Boden et al. 2005; Burgasser 2007).

The TW Hydrae association (TWA) is the first co-moving group of young $(\leq 100 \mathrm{Myr})$, nearby $(\leq 100 \mathrm{pc})$ stars, to be identified near the Sun (Kastner et al. 1997). Ideal observational niche for studing stellar and planetary formation, TWA was actually the tip of an iceberg composed of hundreds of young stars, spread in different groups, which were identified during the last decade (Zuckerman \& Song 2004; Torres et al. 2008). TWA counts now 27 members covering a mass regime from intermediate-mass stars to planetary mass objects (Chauvin et al. 2005). Its $8.3 \pm 0.8 \mathrm{Myr}$ dynamical age was found by a convergence method (de la Reza et al. 2006). Independently, Barrado Y Navascués (2006) estimated an age of $10_{-7}^{+10} \mathrm{Myr}$ from the photometry, the activity, and the lithium depletion. Scholz et al. (2007) show that the association is $9_{-2}^{+8}$ Myr old by comparing rotational velocities with published rotation periods for a subset of stars. Finally, Mentuch et al. (2008) estimate an age of $12 \pm 8 \mathrm{Myr}$ studing lithium depletion in five nearby young associations (hereafter M08).

Song et al. (2003), hereafter S03, identify TWA22 as an M5 member of TWA. The strong Li $\lambda 6708 \AA$ feature supported the extreme youth of this member. Later, Mamajek (2005) question the membership of TWA22 from a kinematic study of TW Hydrae members. Finally, Song et al. (2006) discuss the Mamajek (2005) results that appeared to disagree with the very strong lithium line of the source. The proximity $(17.5 \pm 0.2 \mathrm{pc}$, see Teixeira et al. 2009) and the reported youth of TWA22 by S03 made it consequently a potential target for detecting companions at small orbital radii.

In our program for detecting companions in young associations using the adaptive-optic (AO) assisted imager NACO, we resolved TWA22 as a tight $(\sim 100$ mas $)$ binary. With a projected physical separation of $1.76 \pm 0.10 \mathrm{AU}$ (see Fig. 1), this system offered a unique opportunity to measure its dynamical mass and to possibly test the evolutionary model predictions at young ages using combined photometric and spectroscopic observations.

We report here the discovery of the TWA22 binarity and the results of a dedicated 4 years observing program, using combined imaging and 3D-spectroscopy with AO. The purpose was to measure the dynamical mass of TWA22 AB and to characterize the physical properties of the individual components. In Sect. 2, we describe our AO observations with the VLT/NACO imager and with the VLT/SINFONI integral field spectrograph. The associated data reduction and spectral extraction techniques are explained in Sect. 3. In Sect. 4, we present our orbital solutions and our spectral analysis. In Sect. 5 we compare and discuss the evolutionary model predictions associated to our dynamical mass measurement with the physical properties (surface gravity, temperature, and luminosity) derived from our photometric and spectroscopic observations. This leads us to discuss the membership of TWA22 AB to the TW Hydrae association, the multiplicty of the system, and a possible underestimation of the mass predicted by evolutionary models for young stars close to the substellar boundary.

\section{Observations}

\subsection{VLT/NACO Observations}

TWA22 AB was observed at the $8.2 \mathrm{~m}$ VLT UT4 Yepun with the Nasmyth Adaptive Optics (AO) System NAOS (Rousset et al. 2000) coupled to the high-resolution near-IR camera CONICA (Lenzen et al. 1998). NAOS and CONICA (NACO) resolved the system as a tight binary for the first time on March 5, 2004. Follow-up observations were conducted during four years from early 2004 to end 2007. To image TWA22 AB, we used the narrow band filters: $N B \_1.24\left(\lambda_{0}=1.237 \mu \mathrm{m}, F W H M=0.015 \mu \mathrm{m}\right)$, $N B \_1.75\left(\lambda_{0}=1.748 \mu \mathrm{m}, F W H M=0.026 \mu \mathrm{m}\right)$, and $N B \_2.17$ $\left(\lambda_{0}=2.166 \mu \mathrm{m}, F W H M=0.023 \mu \mathrm{m}\right)$. The broad band filters $J\left(\lambda_{0}=1.27 \mu \mathrm{m}, F W H M=0.25 \mu \mathrm{m}\right), H\left(\lambda_{0}=1.66 \mu \mathrm{m}\right.$, $F W H M=0.33 \mu \mathrm{m})$ and $K_{\mathrm{s}}\left(\lambda_{0}=2.18 \mu \mathrm{m}, F W H M=0.35 \mu \mathrm{m}\right)$ were also used coupled to a neutral density (attenuation factor of 80). CONICA was used with the S13 and S27 cameras to Nyquist-sample the PSF depending on the selected filter. The data were recorded under seeing that ranged from $0.6^{\prime \prime}$ to $1.5^{\prime \prime}$ (see Table 1). TWA22 AB was bright enough in the visible to be used by NAOS for wavefront analysis. For each observation period, dithering around the object in $J, H$, and $K_{\mathrm{s}}$ bands combined with nodding were needed to run a good sky estimation during the data reduction process (see part 2.2). PSF references were observed at different airmasses with identical setups. The $\Theta_{1}$ Ori C astrometric field (McCaughrean \& Stauffer 1994) was observed at each epoch to calibrate the detector platescale and orientation whenever necessary. The results are reported in Table 2.

\subsection{VLT/SINFONI observations}

The SINFONI instrument (Spectrograph for INtegral Field Observations in the Near Infrared), located at the Cassegrain focus of the VLT UT4 Yepun, was used to observe TWA22 AB between February 9 and 13, 2007. SINFONI includes an integral field spectrometer SPIFFI (SPectrograph for Infrared Faint Field Imaging, Eisenhauer et al. 2003), operating in the nearinfrared $(1.1-2.45 \mu \mathrm{m})$. SPIFFI is assisted with the 60 actuators Multi-Applications Curvature Adaptive Optic system MACAO (Bonnet et al. 2003). We used the small SPIFFI field of view of $0.8^{\prime \prime} \times 0.8^{\prime \prime}$ corresponding to a plate scale of 25 mas per pixel to Nyquist-sample the SINFONI AO corrected PSF. The field of view is optically sliced into 32 horizontal slitlets that sample the horizontal spatial direction and that are rearranged to form a pseudo-long slit. Once dispersed by the grating on the $2048 \times 2048$ SPIFFI detector, each slitlet of 64 pixels width (spatial direction) corresponds to 64 spectra of 2048 pixels long (spectral direction). The 2048 independent spectra on the detector are reorganized during the reduction process in a datacube 
Table 1. Observing log.

\begin{tabular}{|c|c|c|c|c|c|c|c|c|c|c|}
\hline UT Date & Name & Instrument & Mode & $\begin{array}{c}\begin{array}{c}\text { Filter } \\
\text { (grism) }\end{array}\end{array}$ & Camera & Airmass & $\begin{array}{l}\text { Seeing } \\
(\operatorname{arcsec})\end{array}$ & $\begin{array}{l}\mathrm{EC}^{a} \\
(\%)\end{array}$ & $\begin{array}{l}\text { Exp. } \\
\text { time } \\
\text { (s) }\end{array}$ & Note \\
\hline $2004 / 03 / 05$ & TWA22AB & NACO & imaging & $N B \_2.17$ & S27 & 1.16 & 1.23 & 16.5 & 20 & \\
\hline 2004/03/05 & TWA22AB & NACO & imaging & $N B \_1.24$ & S13 & 1.15 & 1.40 & 27.7 & 120 & \\
\hline 2004/04/27 & TWA22AB & NACO & imaging & $N B \_1.75$ & S27 & 1.16 & 0.84 & 48.2 & 6 & \\
\hline 2005/05/06 & TWA22AB & NACO & imaging & $H$ & S13 & 1.15 & 0.72 & 12.5 & 5 & \\
\hline 2006/01/08 & TWA22AB & NACO & imaging & $J$ & S13 & 1.16 & 0.84 & 17.5 & 50 & \\
\hline 2006/01/08 & TWA22AB & $\mathrm{NACO}$ & imaging & $H$ & S13 & 1.17 & 0.65 & 17.4 & 50 & \\
\hline $2006 / 02 / 26$ & TWA22AB & NACO & imaging & $H$ & S13 & 1.15 & 1.10 & 32.1 & 150 & \\
\hline 2006/02/26 & TWA22AB & $\mathrm{NACO}$ & imaging & $K_{s}$ & S27 & 1.15 & 1.50 & 10.2 & 50 & \\
\hline 2007/03/06 & TWA22AB & NACO & imaging & $H$ & S13 & 1.15 & 0.60 & 45.7 & 103.5 & \\
\hline 2007/12/04 & TWA22AB & $\mathrm{NACO}$ & imaging & $H$ & S13 & 1.28 & 1.00 & 34.4 & 150 & \\
\hline $2007 / 12 / 26$ & TWA22AB & NACO & imaging & $H$ & S13 & 1.16 & 0.90 & 39.9 & 150 & \\
\hline 2007/02/12 & TWA22AB & SINFONI & spectroscopy & $J(2000)$ & 25 & 1.18 & 1.56 & 12.1 & 1080 & \\
\hline 2007/02/13 & TWA22AB & SINFONI & spectroscopy & $J(2000)$ & 25 & 1.16 & 0.98 & 18.5 & 1080 & \\
\hline 2007/02/13 & HIP049201 & SINFONI & spectroscopy & $J(2000)$ & 25 & 1.13 & 0.86 & 27.0 & 20 & Telluric Std \\
\hline 2007/02/13 & HIP038858 & SINFONI & spectroscopy & $J(2000)$ & 25 & 1.14 & 0.78 & 28.5 & 20 & Telluric Std \\
\hline 2007/02/12 & HIP035208 & SINFONI & spectroscopy & $J(2000)$ & 25 & 1.17 & 1.17 & 15.2 & 60 & Telluric Std \\
\hline 2007/02/11 & TWA22AB & SINFONI & spectroscopy & $H+K(1500)$ & 25 & 1.16 & 0.89 & 29.5 & 960 & \\
\hline 2007/02/09 & TWA22AB & SINFONI & spectroscopy & $H+K(1500)$ & 25 & 1.52 & 1.00 & 17.2 & 960 & \\
\hline 2007/02/11 & HIP052202 & SINFONI & spectroscopy & $H+K(1500)$ & 25 & 1.19 & 0.93 & 24.7 & 20 & Telluric Std \\
\hline 2007/02/09 & HIP052202 & SINFONI & spectroscopy & $H+K(1500)$ & 25 & 1.55 & 1.22 & 14.0 & 40 & Telluric Std \\
\hline
\end{tabular}

${ }^{a}$ Corresponds to the mean strehl ratio for the spectroscopic observations.

Table 2. Mean plate scale and detector orientation for our different observing NACO runs.

\begin{tabular}{cccc}
\hline \hline UT Date & Camera & Plate Scale & $\begin{array}{c}\text { Orientation of true north } \\
\text { east of the vertical } \\
\text { (o) }\end{array}$ \\
\hline $2004 / 03 / 05$ & S27 & $27.01 \pm 0.05$ & $-0.18 \pm 0.19$ \\
$2004 / 04 / 27$ & S27 & $27.01 \pm 0.10$ & $0.08 \pm 0.20$ \\
$2005 / 05 / 06$ & S13 & $13.25 \pm 0.08$ & $-0.02 \pm 0.10$ \\
$2006 / 01 / 08$ & S13 & $13.26 \pm 0.08$ & $0.18 \pm 0.10$ \\
$2006 / 02 / 26$ & S13 & $13.25 \pm 0.06$ & $0.19 \pm 0.11$ \\
$2007 / 03 / 06$ & S13 & $13.28 \pm 0.10$ & $0.08 \pm 0.15$ \\
$2007 / 12 / 04$ & S13 & $13.29 \pm 0.12$ & $-0.21 \pm 0.17$ \\
$2007 / 12 / 26$ & S13 & $13.29 \pm 0.12$ & $-0.21 \pm 0.17$ \\
\hline
\end{tabular}

that contains the spatial $(X, Y)$ and the spectral $(Z)$ information. The cube is resampled in the vertical dimension $(Y)$ to have the same number of pixels as in $X$.

To cover the full spectral range between 1.1 to $2.45 \mu \mathrm{m}$, individual integrations times of $90 \mathrm{~s}$ were necessary for imaging the system in the $J$ band $(1.1-1.4 \mu \mathrm{m}, R=2000)$ and $20 \mathrm{~s}$ in the $H+K$ band $(1.45-2.45 \mu \mathrm{m}, R=1500)$. For each band, dithering around the object was used to increase the field of view and to suppress residual bad pixels, leading to a total observing time on target of $\sim 5 \mathrm{~min}$. An additional frame was acquired on the sky to improve our correction. The adaptive optic loop was locked on TWA22 AB itself. Standard stars HIP038858 (B3V), HIP049201 (B2V), HIP035208 (B3V), and HIP052202 (B4V) were observed at similar airmasses to remove the telluric lines (see Table 1).

\section{Data reduction and analysis}

\subsection{High-contrast imaging}

For each observation period, the ESO eclipse reduction software (Devillard 1997) dedicated to AO image processing was used on the complete set of raw images. Eclipse computes bad-pixel detection and interpolation, flat-field correction, and averaging pairs of shifted images with sub-pixel accuracy. The software run sky estimation on object-dithered frames using median filtering through the frame sequence.

A deconvolution algorithm dedicated to the stellar field blurred by the adaptive-optics corrected point spread functions (Veran \& Rigaut 1998) was applied to TWA22 AB images to accurately find the position and the photometry of the companion relative to the primary. The algorithm is based on the minimization in the Fourier domain of a regularized least square objective function using the Levenberg-Marquardt method. We used Nyquist-sampled unsaturated images of standard stars obtained the same night as TWA22 observations with identical setups under various atmospheric conditions. These frames captured the variation in AO corrections. They were used as input point spread functions (PSF) to estimate the deconvolution process error. The IDL Starfinder ${ }^{1}$ PSF fitting package (Diolaiti et al. 2000) confirmed these results.

\subsection{Integral field spectroscopy}

We used the SINFONI data reduction pipeline (1.7.1 version, see Modigliani et al. 2007) for raw data processing. The pipeline carries out cube reconstruction from raw detector images. The flagging of hot and non linear pixels is executed in a similar way to NACO images. The distortion and wavelength scale are calibrated on the entire detector using arc-lamp frames. Slitlet distances are accurately measured with north-south scanning of the detector illuminated with an optical fiber. In the case of standard stars observation, object-sky frame pairs are subtracted, flatfielded, and corrected from bad pixels and distortions. Datacubes are finally reconstructed from clean science images and merged in a master cube. Spectra of standard stars cleaned from stellar lines are finally used to correct the TWA22 AB spectra from telluric absorptions.

\footnotetext{
1 IDL procedures can be downloaded at http://www . bo. astro.it/ giangi/StarFinder/index.htm.
} 
Table 3. Relative positions and contrasts of TWA22 A and B, with magnitude differences given in the NACO photometric system.

\begin{tabular}{cccccc}
\hline \hline UT Date & Filter & Camera & $\begin{array}{c}\Delta \alpha_{\mathrm{J} 2000} \\
(\mathrm{mas})\end{array}$ & $\begin{array}{c}\Delta \delta_{\mathrm{J} 2000} \\
(\mathrm{mas})\end{array}$ & $\begin{array}{c}\Delta m \\
(\mathrm{mag})\end{array}$ \\
\hline $2004 / 03 / 05$ & $N B 2.17$ & $\mathrm{~S} 27$ & $99 \pm 3$ & $-17 \pm 3$ & $0.33 \pm 0.12$ \\
& $N B 1.24$ & $\mathrm{~S} 13$ & & & $0.37 \pm 0.10$ \\
$2004 / 04 / 27$ & $N B 1.75$ & $\mathrm{~S} 13$ & $98 \pm 6$ & $-36 \pm 6$ & $0.41 \pm 0.10$ \\
$2005 / 05 / 06$ & $H-N D$ & $\mathrm{~S} 13$ & $15 \pm 3$ & $-89 \pm 3$ & $0.66 \pm 0.05$ \\
$2006 / 01 / 08$ & $J$ & $\mathrm{~S} 13$ & & & $0.40 \pm 0.04$ \\
& $H$ & $\mathrm{~S} 13$ & $-68 \pm 2$ & $-49 \pm 2$ & $0.54 \pm 0.05$ \\
$2006 / 02 / 26$ & $H$ & $\mathrm{~S} 13$ & $-74 \pm 3$ & $-30 \pm 3$ & $0.54 \pm 0.05$ \\
& $K_{s}$ & $\mathrm{~S} 27$ & & & $0.46 \pm 0.18$ \\
$2007 / 03 / 06$ & $H$ & $\mathrm{~S} 13$ & $-57 \pm 4$ & $80 \pm 2$ & $0.49 \pm 0.03$ \\
$2007 / 12 / 04$ & $H$ & $\mathrm{~S} 13$ & $19 \pm 3$ & $98 \pm 3$ & $0.52 \pm 0.10$ \\
$2007 / 12 / 26$ & $H$ & $\mathrm{~S} 13$ & $26 \pm 3$ & $97 \pm 3$ & $0.53 \pm 0.10$ \\
\hline
\end{tabular}

TWA22 A and B are centered and oriented horizontally in the $J$ and $H+K$ master cubes with a field of view of $1.1^{\prime \prime} \times 1.1^{\prime \prime}$. Atmospheric refraction induces different sources positions for different wavelengths within the instrument field of view and increases with airmass. Combined with the small SINFONI field of view, this produces differential flux losses that were noticed in the bright standard-star datacubes. This effect remains limited for TWA22. The cubes of February 11, 2007 appear to have some spectra contaminated by flux oscillations of a few ADUs. These oscillations were not negligible and blurred $\mathrm{CO}$ bands at $2.3 \mu \mathrm{m}$. They are present along the dispersion axis in the raw detector images of both HIP052202 and TWA22. Their amplitudes do not remain constant in time but follow a 15.3 pixel period. We then filtered partially this contribution on each individual image in the Fourier space using a pass-band function. The origin of the problem is likely to be related to $50 \mathrm{~Hz}$ pick-up noise.

We used a modified version (Dumas et al. 2001) of the CLEAN algorithm (Högbom 1974; Schwartz 1978) to extract the flux of TWA22 A and B separately in each monochromatic image contained in the datacubes. The standard star is used for initial PSF-references. Once scaled to match the TWA22 A maximum at the primary position and for all wavelengths, the PSF is subtracted from the TWA22 AB datacube. The sequence is repeated to model the secondary contribution, cleaned from the primary wings, and to provide a new PSF-reference. After a few iterations minimizing the final quadratic residual datacube, the spectra of each individual component are extracted.

The algorithm was first adapted to work on cube images. Unfortunately, the difference of sampling between the $X$ and $Y$ directions limited the subpixel shift accuracy. We therefore collapsed the cube along the $Y$-axis to obtain the flux profile along the $X$ direction. We chose to duplicate the primary flux profile for the PSF model. The algorithm converged in a few iterations and produced extracted spectra in $J$ and $H+K$ with an extraction error less than $5 \%$. The extracted spectra were divided by standard star spectra corrected from intrinsic features and multiplied by a black body spectrum at the standard star temperature. The SINFONI pipeline coefficients were used for wavelength calibration.

\section{Results}

\subsection{Astrometry, orbit and dynamical mass}

The relative positions of TWA $22 \mathrm{~A}$ and $\mathrm{B}$ (B with respect to A) at all observation epochs are reported in Table 3. The data allows determination of the mutual orbit of the binary. We define a cartesian referential frame $(O, X, Y, Z)$ where $X$ points towards the north, $Y$ toward the east, and $Z$ toward the Earth. The $(O X Y)$ plane thus corresponds to the plane of the sky. Then in a Keplerian formalism, the projected position $(x \equiv \Delta \delta, y \equiv \Delta \alpha)$ of the binary onto the plane of the sky reads

$$
\begin{aligned}
x= & \frac{a}{d}(\cos u-e)(\cos \omega \cos \Omega-\sin \omega \cos i \sin \Omega) \\
& +\frac{a}{d} \sqrt{1-e^{2}} \sin u(-\sin \omega \cos \Omega-\cos \omega \cos i \sin \Omega), \\
y= & \frac{a}{d}(\cos u-e)(\cos \omega \sin \Omega+\sin \omega \cos i \cos \Omega) \\
& +\frac{a}{d} \sqrt{1-e^{2}} \sin u(-\sin \omega \sin \Omega+\cos \omega \cos \Omega \cos i),
\end{aligned}
$$

where $a$ is the semi-major axis of the orbit (in AU), $d$ the distance of the binary (in pc), $e$ the eccentricity, $i$ the inclination, $\Omega$ the longitude of ascending node (counted from north towards east), $\omega$ the argument of periastron, and $u$ is the eccentric anomaly that describes the current location of the binary along its orbit. The value of $u$ is related to the time $t$ by the classical Kepler's equation

$\frac{2 \pi}{T}\left(t-t_{\mathrm{p}}\right)=u-e \sin u$

where $T$ is the orbital period and $t_{\mathrm{p}}$ the time reference for periastron passage. Once the distance $d$ is known $(17.5 \mathrm{pc}$, Teixeira et al. 2009), the fit of the observational data allows determinating the 7 parameters $T, a, e, i, \Omega, \omega$, and $t_{\mathrm{p}}$. Then Kepler's third law leads to the total mass $M$.

The fit is performed via a Levenberg-Marquardt $\chi^{2}$ minimizing algorithm. In practice, instead of $(e, i, \Omega, \omega)$, the equations are solved for the classical variables

$$
\begin{aligned}
& k=e \cos (\Omega+\omega) \quad q=\sin \frac{i}{2} \cos \Omega \\
& h=e \sin (\Omega+\omega) \quad p=\sin \frac{i}{2} \sin \Omega,
\end{aligned}
$$

which avoids singularities towards small eccentricities and inclinations. The uncertainties on the fitted parameters are estimated from the resulting covariance matrix at the end of the fit procedure.

Levenberg-Marquardt is an interative gradient method for converging towards a mininum of the $\chi^{2}$ function. Depending on the starting guess point, many local minima can be found. In the present case, all the attemps we made (by letting the starting point vary) converge towards the same solution that is listed in Table 4 and viewed in projection onto the plane of the sky in Fig. 2. The available astrometric data set appears to cover almost one complete orbital period with a good sampling of the periastron passage. We are thus confident in our fitted solution. The orbit then appears slighly eccentric $(e \simeq 0.1)$ and viewed close to pole-on $i \simeq 27^{\circ}$ from the Earth.

\subsection{Photometry}

Table 3 summarizes the magnitude differences between TWA22 A and B, measured with NACO at different wavelengths. Taking the filter tranformations between NACO and 2MASS and the photometry of the unresolved system given from the 2MASS Survey (Cutri et al. 2003) into account, we derived the apparent $J H K$ magnitudes of each component (see Table 5). Observations under bad seeing conditions were excluded. Based on an accurate distance $(17.5 \pm 0.2 \mathrm{pc})$ determination 
Table 4. TWA22 B orbital parameters as determined from the fit of the astrometric data (see text for the definition of the parameters) with the reduced $\chi^{2}$ of the fit.

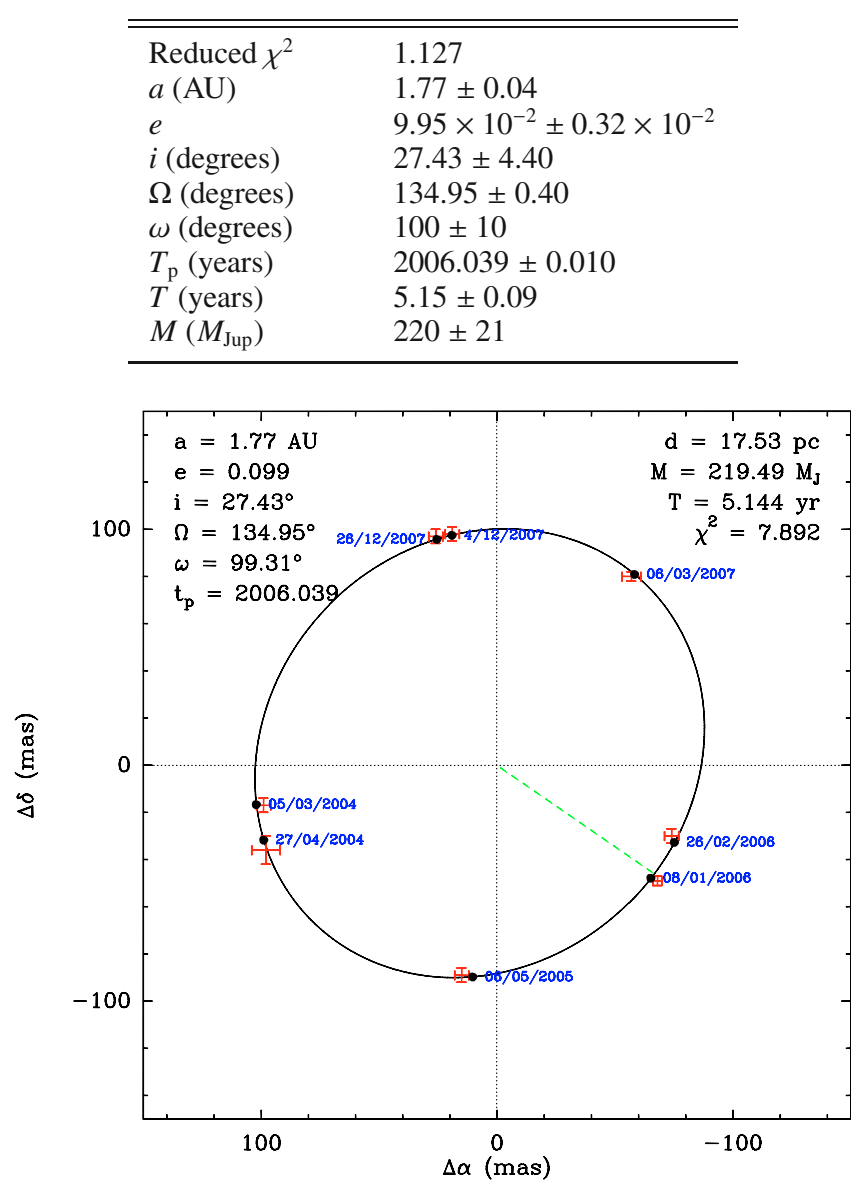

Fig. 2. Orbital fit of the relative positions of TWA22 AB observed from March 2004 to December 2007, as projected onto the plane of the sky. The crosses represent the observational data with their error bars, the solid line is the fitted projected orbit, and the dots correspond to the predicted positions of the model at the times of the observations. The dashed line sketches the projected direction of the periastron of the orbit. On January 8, 2006, the binary was actually very close to periastron.

(Teixeira et al. 2009), the absolute magnitudes were also derived. Since TWA22 is a young mid-M system, we monitored its photometric variations in the $\mathrm{H}$ band. We only noticed a 0.05 variation of the total magnitude of the system over time. This variation is reported in the error bars on our photometry in Table 5.

Reported in a color $(J-K)$-magnitude $\left(M_{K \mathrm{~s}}\right)$ diagram, the TWA22 A and B photometry can be compared with the photometry of $\mathrm{M}$ dwarfs (see Fig. 3) of the young, nearby associations TW Hydrae ( $\sim 8 \mathrm{Myr}), \beta$ Pictoris ( $\sim 12 \mathrm{Myr})$, TucanaHorologium ( $30 \mathrm{Myr})$ and AB Doradus ( $70 \mathrm{Myr})$. At a given age, early-type $\mathrm{M}$ dwarfs are bluer and more luminous than latetype $\mathrm{M}$ dwarfs in the $K$ band. At a given spectral type (i.e. temperature), the objects go fainter with age. Although agedependent, the near-infrared photometry of TWA22 A and B appears compatible for both components with what is expected for young mid-M dwarfs but does not allow an age estimation for the binary. Predictions of evolutionary models of (Baraffe et al. 1998, named NEXTGEN) are also given at these young ages. The NEXTGEN tracks appears bluer than 10 Myr old mid-M
Table 5. TWA22 A and B individual magnitudes converted into the 2MASS system.

\begin{tabular}{ccccc}
\hline \hline Band & $\begin{array}{c}m_{A} \\
(\mathrm{mag})\end{array}$ & $\begin{array}{c}m_{B} \\
(\mathrm{mag})\end{array}$ & $\begin{array}{c}M_{A} \\
(\mathrm{mag})\end{array}$ & $\begin{array}{c}M_{B} \\
(\mathrm{mag})\end{array}$ \\
\hline$J$ & $9.12 \pm 0.10$ & $9.52 \pm 0.11$ & $7.90 \pm 0.13$ & $8.30 \pm 0.14$ \\
$H$ & $8.61 \pm 0.15$ & $9.12 \pm 0.15$ & $7.39 \pm 0.18$ & $7.90 \pm 0.18$ \\
$K_{\mathrm{s}}$ & $8.24 \pm 0.19$ & $8.70 \pm 0.25$ & $7.02 \pm 0.23$ & $7.48 \pm 0.28$ \\
\hline
\end{tabular}

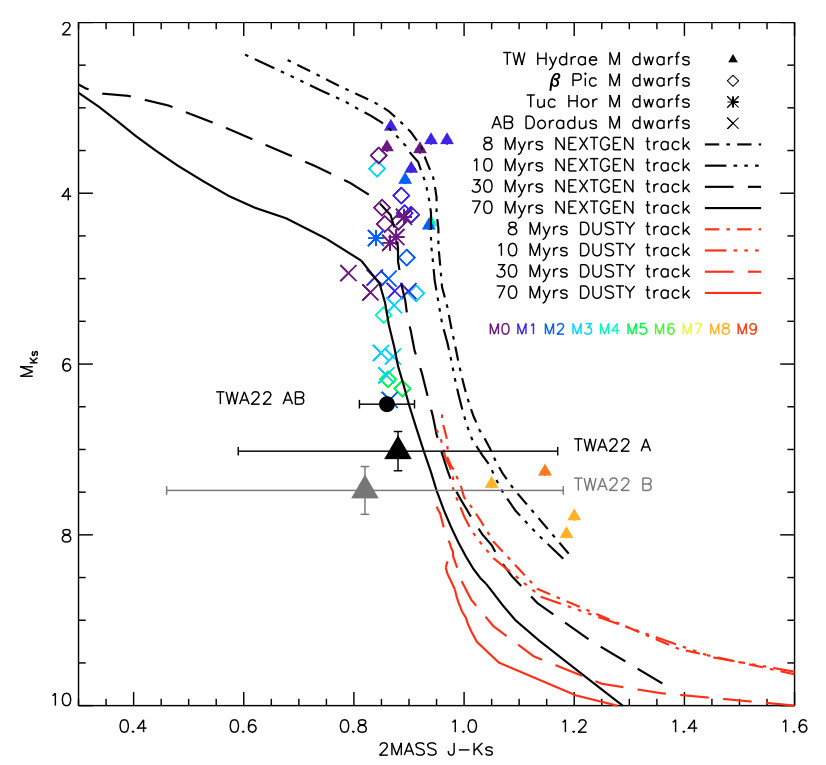

Fig. 3. Color $(J-K)$ - magnitude $\left(M_{K s}\right)$ diagram of TWA22 A and B compared with the photometry of young M dwarfs members of the TW Hydrae (age $=8 \mathrm{Myr}$, filled triangles), $\beta$ Pictoris (age $=10 \mathrm{Myr}$, diamonds), Tuc-Hor (age = $30 \mathrm{Myr}$, stars), and AB Dor (age $=70 \mathrm{Myr}$, crosses) associations. Distances and spectral types were taken from Torres et al. (2008) except for the members of the TW Hydrae moving group (Mamajek 2005). Typical error bars are 0.03 for $M_{K}$ and 0.08 for $J-K_{\mathrm{s}}$. NEXTGEN (Baraffe et al. 1998) 8 Myr (dashed-dotted black line), 10 Myr (long-dashed-dotted black line), 30 Myr (long-dashed black line) and $70 \mathrm{Myr}$ isochrones (full black line) are overplotted. DUSTY tracks (Chabrier et al. 2000) at identical ages were added (red lines). These tracks were shifted by 0.2 mag to redder $J-K \mathrm{~s}$. This artificially compensates for the remaining incompleteness, at relatively warm temperatures, in the AMES linelists used in DUSTY atmospheric models (Mohanty et al. 2007). The NEXTGEN tracks also appear bluer than 10 Myr old mid-M dwarfs by $\sim 0.2 \mathrm{mag}$, which might be related to a partial representation of their spectral energy distribution. At a given age, early-type $\mathrm{M}$ dwarfs (violet to green points) are bluer and more luminous than late-type $\mathrm{M}$ dwarfs (green to red points) in the $K$ band. At a given spectral type (i.e. temperature), the objects go fainter with age. The photometry is compatible with the one of young mid-M dwarfs. The unresolved 2MASS photometry is plotted for comparison (black dot).

dwarfs by $\sim 0.2 \mathrm{mag}$, which might be related to a partial representation of their spectral energy distribution. At relatively warm temperatures, the remaining incompleteness in the AMES linelists used in DUSTY atmospheric models (Mohanty et al. 2007) lead to $J-K$ s colors redder than those observed for lateM dwarfs. The DUSTY evolutionary tracks were then shifted by 0.2 mag to redder $J-K \mathrm{~s}$. We provide in the following an improved estimation of the spectral type of our targets, using our spectroscopic data. 


\subsection{Spectroscopic analysis}

\subsubsection{Line identification}

To identify the numerous spectral features in the TWA22 A and B spectra between 1.10 to $2.45 \mu \mathrm{m}$, the spectra were compared with an homogeneous medium resolution $\left(R_{\lambda} \sim 2000\right)$ sequence of field dwarfs from Cushing et al. (2005) (hereafter C05; see Figs. 4-6). The TWA22 A and B spectra appear very similar.

In $J$-band, both TWA22 A and B spectra are dominated by the strong $\mathrm{Na}$ I doublet at $1.138 \mu \mathrm{m}$, the deep $\mathrm{K}$ I lines at 1.169 , $1.177,1.243$, and $1.253 \mu \mathrm{m}$, and the presence of a broad $\mathrm{H}_{2} \mathrm{O}$ absorption from 1.32 to $1.35 \mu \mathrm{m}$. Fe I absorptions are also detected around $1.170 \mu \mathrm{m}$. One is blended with the $1.177 \mu \mathrm{m}$ K I line. We notice additional broad $\mathrm{FeH}$ absorptions around $1.20 \mu \mathrm{m}$ and $1.24 \mu \mathrm{m}$ compatible with what is expected for mid-M dwarfs as well as the presence of the weak Q-branch at $1.22 \mu \mathrm{m}$. Finally, the $\mathrm{Al}$ I doublet at $1.313 \mu \mathrm{m}$ is detected. This doublet is expected to disappear at the M-L transition.

In the $\mathrm{H}$-band, the spectra are affected by $\mathrm{H}_{2} \mathrm{O}$ absorptions from 1.45 to $1.52 \mu \mathrm{m}$ and from 1.75 to $1.8 \mu \mathrm{m}$. They exhibit pronounced K I atomic lines at $1.517 \mu \mathrm{m}$, as well as weak doublets of $\mathrm{Mg}$ I at $1.503 \mu \mathrm{m}$ and $\mathrm{Al} \mathrm{I}$ at $1.675 \mu \mathrm{m}$. Weak FeH absorptions are also present. They increase from M5 to the M-L transition Cushing et al. (2003) and their depths are compatible here with those expected for M5 to M7 field dwarfs.

In $K$-band, strong $\mathrm{H}_{2} \mathrm{O}$ absorptions appear from 1.95 to $2.04 \mu \mathrm{m}$ and from 2.3 to $2.45 \mu \mathrm{m}$. They are typical of mid-M to mid-L dwarfs. Strong Ca I features are present from 1.9 to $2.0 \mu \mathrm{m}$. They tend to disappear in the spectra of field dwarfs at the M-L transition. We firmly identify the first overtone of $\mathrm{CO}$ near $2.3 \mu \mathrm{m}$, the rest being affected by the $50 \mathrm{~Hz}$ pick-up noise oscillations mentioned earlier. Additional weak Mn I, Ti I, $\mathrm{Mg}$ I and Si I absorptions are spread over the $J, H$, and $K$ bands. These lines are expected to be rapidly replaced by molecular absorptions for dwarfs later than M5. The $1.106 \mu \mathrm{m}$ band seems to be overlapping $\mathrm{H}_{2} \mathrm{O}$ and $\mathrm{TiO}$ absorptions with increasing depths from early to late $\mathrm{M}$ dwarfs. Finally, the $1.626 \mu \mathrm{m}$ feature corresponds to close OH lines, as noted in Leggett et al. (1996).

To conclude, the features detected over the spectra of TWA22 A and B between 1.1 and $2.45 \mu \mathrm{m}$ suggest that both components have a cool atmosphere, typical of mid to late-M dwarfs.

\subsubsection{Continuum-fitting and spectral indexes}

The continuum of both TWA22 A and B spectra were compared to spectra of field M dwarfs obtained by C05 and McLean et al. (2003), hereafter ML03. Least squares were computed on parts of the spectra free from telluric correction residuals. From $1.10 \mu \mathrm{m}$ to $1.27 \mu \mathrm{m}$, the TWA22 A and B continuums are reproduced by M6 \pm 1 dwarfs. The $H$-band spectra are poorly reproduced visually. Least squares are minimized for M9 dwarfs but with 2 subclasses of uncertainty. Finally, our $K$-band spectra are well-fitted by M5 to M7 dwarfs. From these comparisons, we assign a spectral type M6 \pm 1 to both TWA22 AB components.

Because TWA22 AB is a young system, we tested whether using high surface gravity spectra of old field dwarfs might affect our spectral analysis. Intermediate surface gravity reduces the strength of alkali lines (Lucas et al. 2001; Gorlova et al. 2003; McGovern et al. 2004; Kirkpatrick et al. 2006) and produces triangular shape in $H$-band interpreted as collision-induced absorptions (CIA) of $\mathrm{H}_{2}$. Our spectra were then compared with young (age $\lesssim 8 \mathrm{Myr}$ ) dwarf spectra (Gorlova et al. 2003;

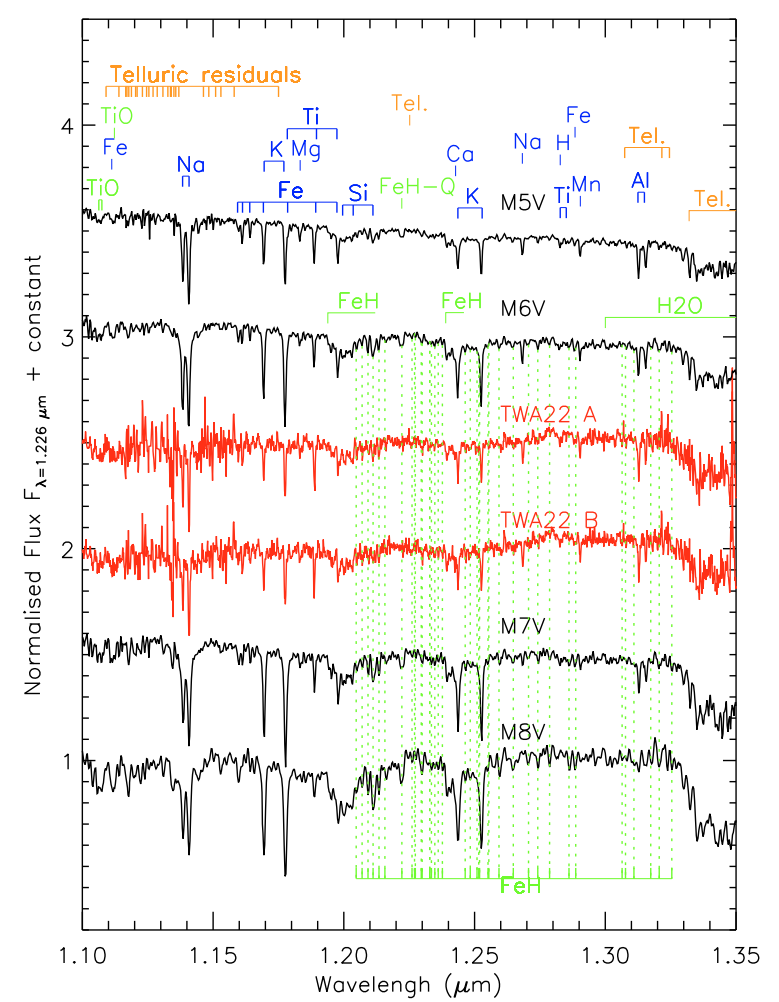

Fig. 4. TWA22 A and B spectra compared to spectra of M5 to M8 dwarfs. The M6 dwarf spectrum reproduces the $J$ bands of TWA22 A and B. However, our spectra seem to have a slightly redder slope. We report identified atomic features (blue). Molecular absorptions ( $\mathrm{FeH}$ bands were identified by Cushing et al. 2003) are flagged in green and telluric residuals in orange. Atomic absorptions are indicated in blue.

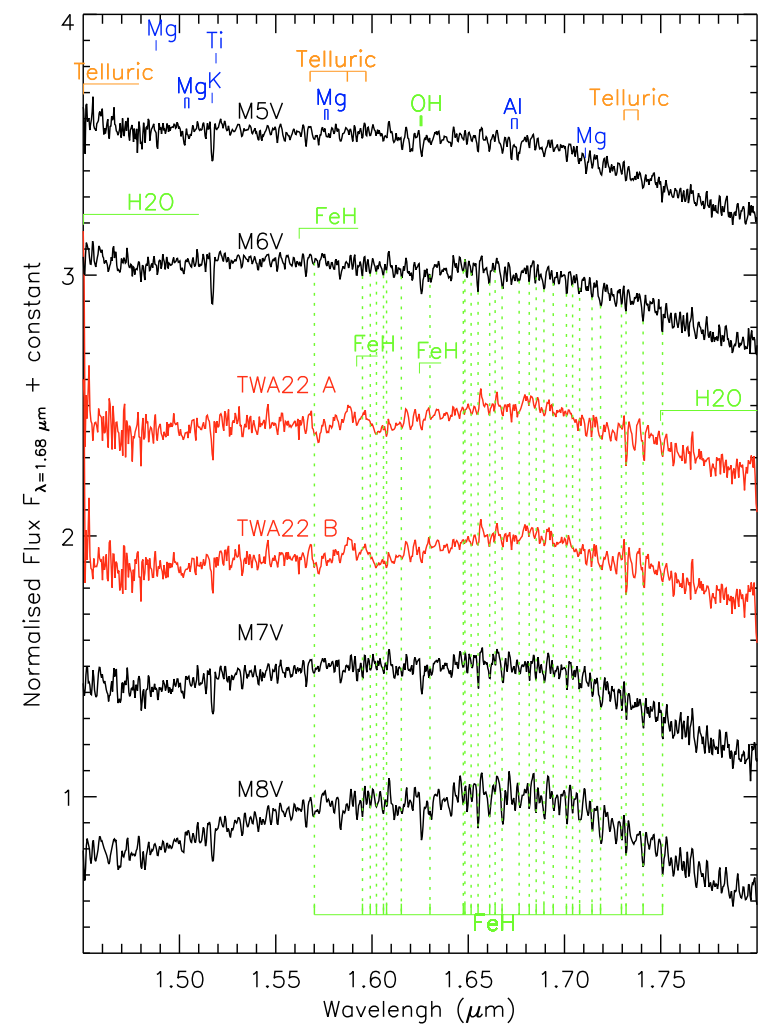

Fig. 5. Same as Fig. 4 but for $H$ band. In this case, differences between M6V field dwarf spectrum and TWA22 spectra are important. This could arise from low gravity or flux losses introduced either in the standard star datacube and during the extraction process. 


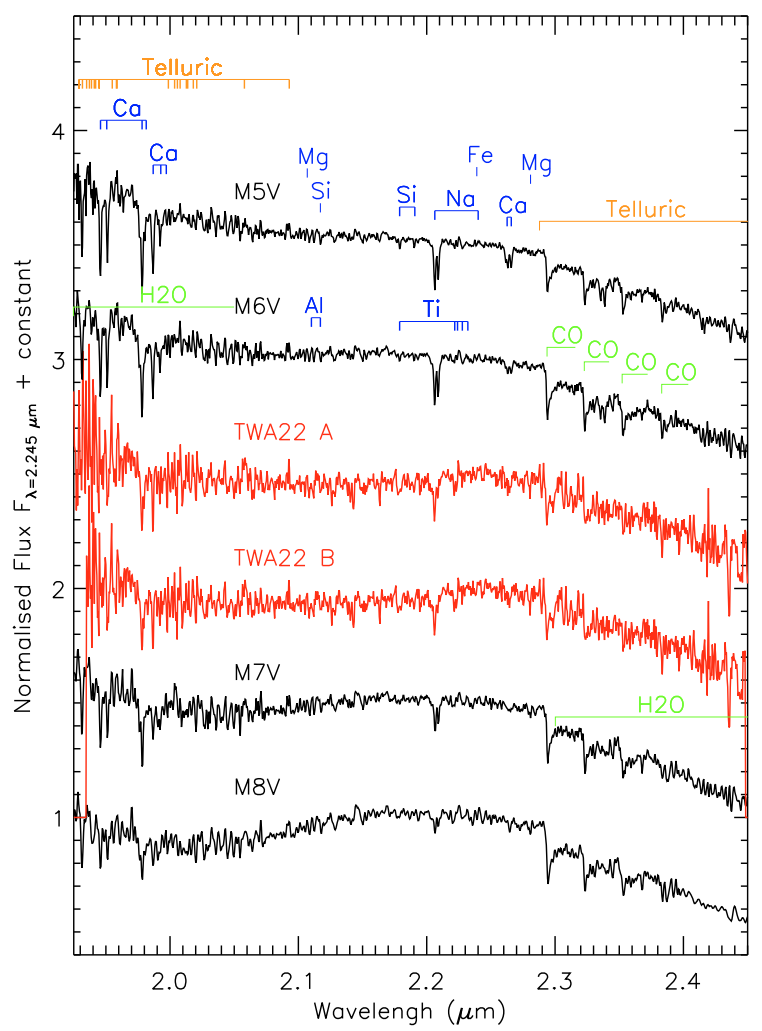

Fig. 6. Same as Fig. 4 but for the $K$ band. Spectra look like an M6V field dwarf.

Slesnick et al. 2004; Lodieu et al. 2008) at identical resolution (spectra were convolved with a Gaussian if necessary) in the $J$ and $K$ bands (see Figs. 7 and 8 ). They are mostly similar to M5, M5.5, M6, and M7 dwarf spectra, and consistent with the continuum fit obtained with field dwarfs. In both cases, our $J$-band spectra are slightly redder than young and old mid-M dwarfs. Our $H$-band spectra are visually still poorly reproduced by our spectral templates. This could arise from low gravity or flux losses introduced either in the standard star datacube and during the extraction process.

To complete this spectral type determination, spectral indexes developed by ML03 (from $\mathrm{H}_{2} \mathrm{O}$ bands at $1.34 \mu \mathrm{m}\left(\mathrm{H}_{2} \mathrm{OA}\right)$, $1.79 \mu \mathrm{m}\left(\mathrm{H}_{2} \mathrm{OC}\right), 1.96 \mu \mathrm{m}\left(\mathrm{H}_{2} \mathrm{OD}\right)$, and at $1.2 \mu \mathrm{m}$ from the $\mathrm{FeH}$ band) were derived for TWA22 A and B (see Fig. 9). The results were compared to the values computed from the ML03 and C05 spectral libraries of field dwarfs. They were also compared to values derived for young dwarfs (Slesnick et al. 2004; Lodieu et al. 2008, hereafter S04 and L08), to test the sensitivity of these indexes to surface gravity (age). In fact, The $\mathrm{H}_{2} \mathrm{O} \mathrm{D}$ and $\mathrm{FeH}$ indexes values tend to change with age for M5-L2 dwarf, and could disturb our analysis. We then used a mean weight of the individual spectral type estimations from $\mathrm{H}_{2} \mathrm{OA}, \mathrm{H}_{2} \mathrm{OC}$ and the recently defined Allers $\mathrm{H}_{2} \mathrm{O}$ index at $1.55 \mu$ m (see Allers et al. 2007) to infer M5 \pm 1 and M5.1 \pm 1 spectral types for TWA22 $\mathrm{A}$ and $\mathrm{B}$ respectively. These results match the $\mathrm{M} 6 \pm 1.5$ and $\mathrm{M} 5 \pm 1$ values derived for TWA22 A and B from the $\mathrm{H}_{2} \mathrm{OD}$ and $\mathrm{FeH}$ index for the 2 objects. Based on the $K$-band photometry and the associated bolometric corrections of Golimowski et al. (2004), we derived a luminosity of $\log \left(L / L_{\odot}\right)=-2.11 \pm 0.13 \mathrm{dex}$ for TWA22 A and $\log \left(L / L_{\odot}\right)=-2.30 \pm 0.16$ dex TWA22 B. Using the $T_{\text {eff-spectral type conversion scales for intermediate- }}$ gravity objects (Luhman et al. 2003), we find an initial estimation of $T_{\text {eff }}=2990_{-190}^{+135} \mathrm{~K}$ for both components.

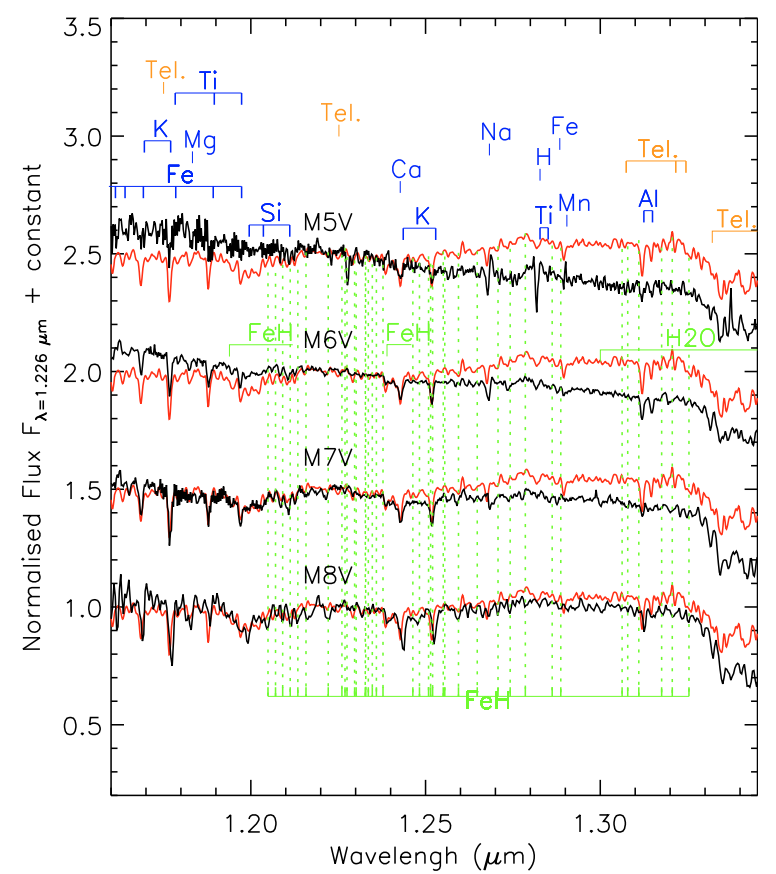

Fig. 7. Comparison of the TWA22 B $J$ band spectrum (red) to spectra (black) of young Upper Sco and Orion nebulae cluster objects (Slesnick et al. 2004; Lodieu et al. 2008). We clearly notice that the TWA22 B spectral slope is redder than that for reference spectra. The TWA22 B spectrum is very similar to those of young M6 and M7 dwarfs. We report identified atomic features (flagged in blue). Molecular absorptions (FeH bands were identified by Cushing et al. 2003) are flagged in green and telluric residuals in orange.

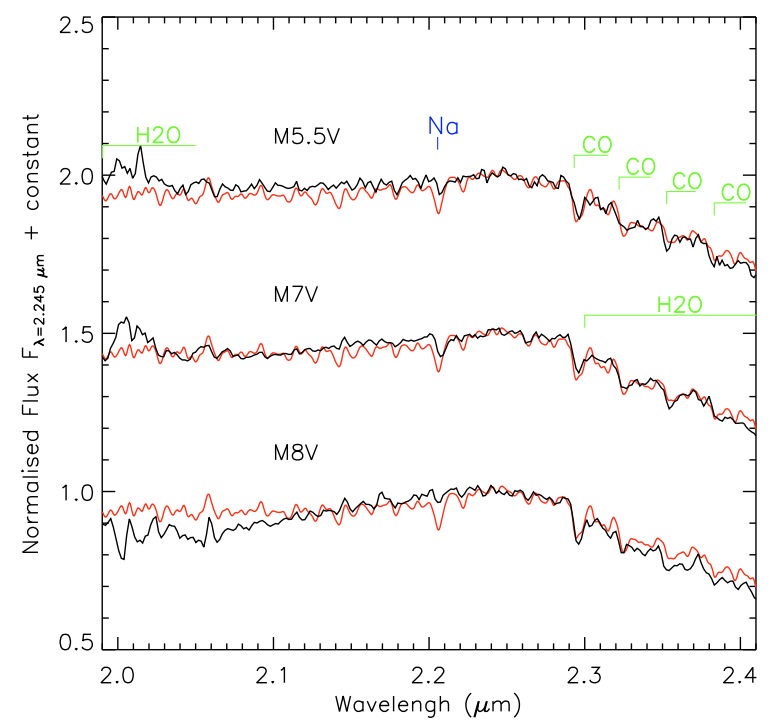

Fig. 8. Comparison of the TWA22 B $K$ band spectrum (red) to spectra of young Upper Sco standards (black) at $R=350$ (Gorlova et al. 2003). The TWA22 B spectrum was convolved with a Gaussian to match the resolution of standard star spectra. Our spectrum is reproduced by spectra of young M5.5 and M7 dwarfs.

\subsubsection{Study of narrow lines}

Depths of many narrow lines were studied to provide additional information on the surface gravity of TWA22 AB, particularly on its age. Following the Sembach \& Savage (1992) method for measuring pseudo-equivalent widths and their associated 


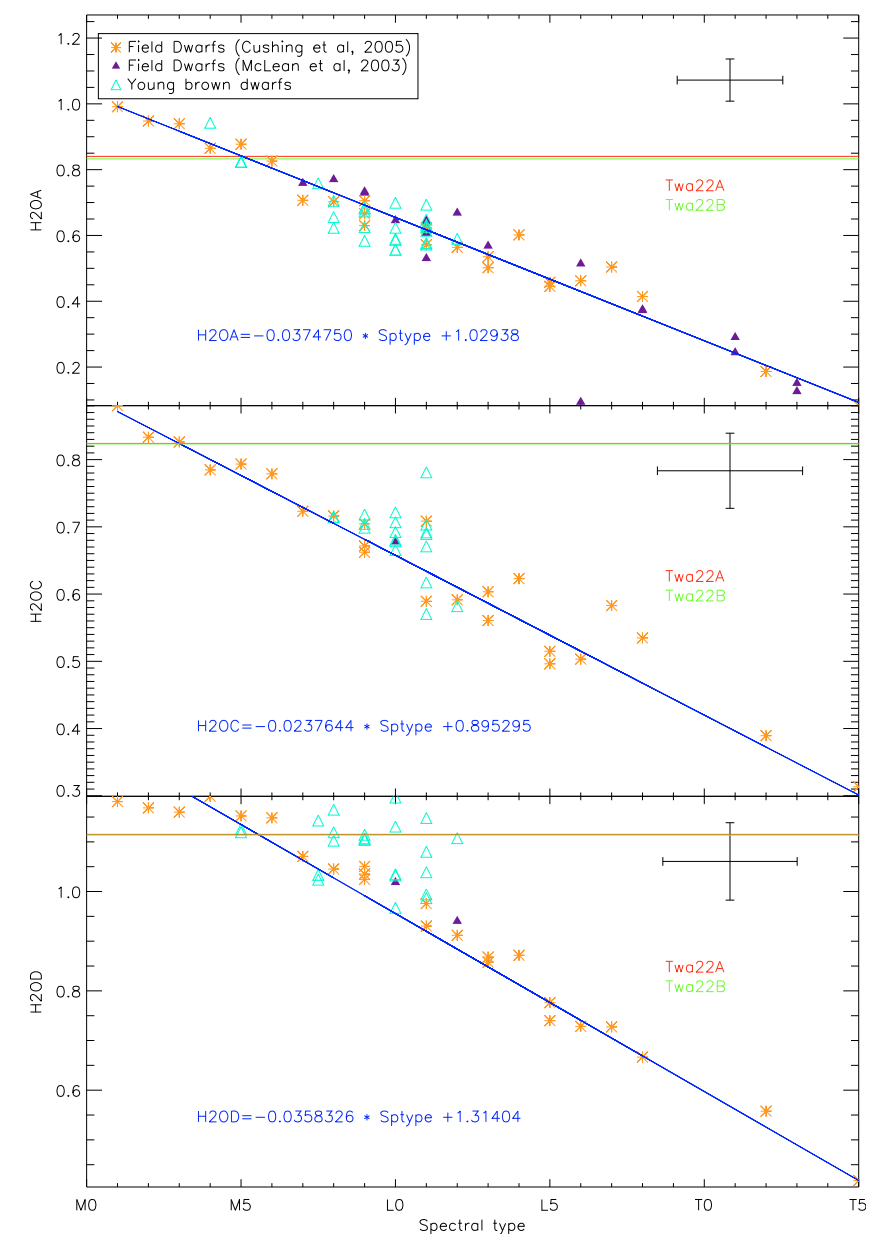

Fig. 9. $\mathrm{H}_{2} \mathrm{O}$ spectral indexes computed on libraries of field (McLean et al. 2003; Cushing et al. 2005) and young dwarfs spectra (Slesnick et al. 2004; Lodieu et al. 2008). Redundancies between libraries have been checked. Young dwarfs M4-L2 dwarfs follow trends of the field dwarfs except for $\mathrm{H}_{2} \mathrm{O}$ D. Using $\mathrm{H}_{2} \mathrm{OA}, \mathrm{H}_{2} \mathrm{OC}$, and the recently defined Allers $\mathrm{H}_{2} \mathrm{O}$ index at $1.55 \mu \mathrm{m}$ (see Allers et al. (2007)), we infer M5 \pm 1 and M5.1 \pm 1 spectral types for TWA22 A and B respectively. In the upper right corner of each plot are reported the $1 \sigma$ errors derived from the residuals to the linear fit.

uncertainties, we derived the equivalent widths of strong atomic lines over the $J, H$, and $K$ bands. They were computed for narrow lines at $1.106 \mu \mathrm{m}\left(\mathrm{TiO}\right.$ and $\left.\mathrm{H}_{2} \mathrm{O}\right), 1.220 \mu \mathrm{m}(\mathrm{FeH}-\mathrm{Q}$ branch), $1.313 \mu \mathrm{m}(\mathrm{Al} \mathrm{I})$, and $1.626 \mu \mathrm{m}(\mathrm{OH})$, and for the $\mathrm{KI}$ doublets at $1.169,1.177,1.243$ and $1.253 \mu \mathrm{m}$. The results were compared with pseudo-equivalent widths of old field dwarfs (C05, ML03) and young Upper Sco dwarfs (S04, L08). The use of both librairies confirmed the strong surface gravity dependency of the $\mathrm{K} \mathrm{I}$ lines, more moderate for the $\mathrm{Al} \mathrm{I}, \mathrm{FeH}$, and $\mathrm{OH}$ lines. Due to the degeneracy in terms of effective temperature and surface gravity, pseudo-equivalent widths alone are not sufficient for a precise spectral type determination of TWA22 AB. They remain, however, compatible with narrow lines depths of young and old dwarfs of spectral types later than M4.

If we now assume a spectral type M6 \pm 1 for both components, pseudo-equivalent widths in the KI lines of TWA22 AB appear intermediate between values found for young and field dwarfs (see Fig. 10). This is confirmed using a visual comparison with an evolutionary sequence of M6 dwarfs composed of the old field dwarf GL 406, the intermediate old companion AB Doc C (Age 75 Myr, M5.5, Close et al. 2007),

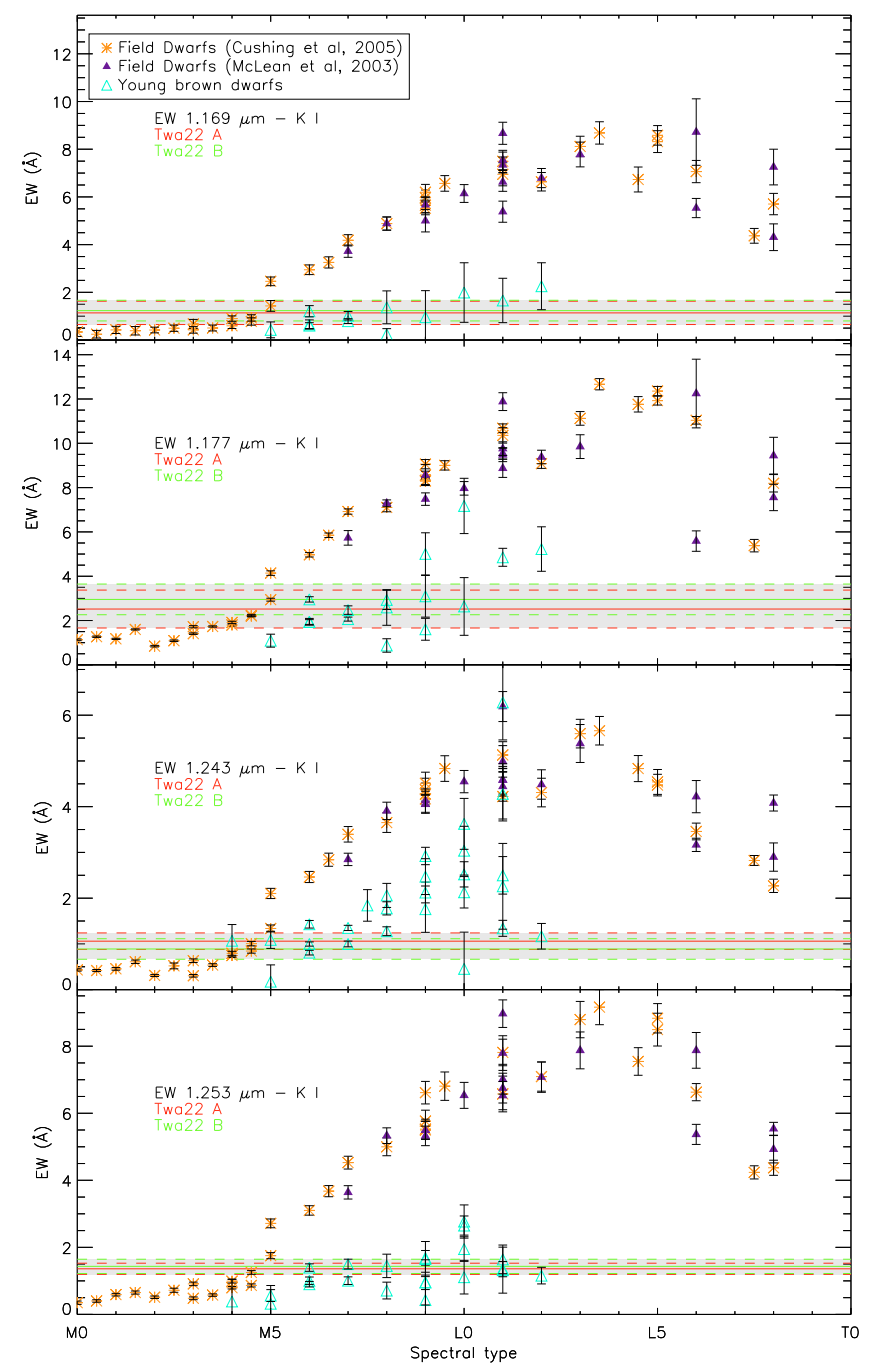

Fig. 10. Equivalent widths in the KI lines computed on libraries of young and field dwarf spectra. Young dwarf spectra have weak K I lines and therefore low equivalent widths compared to field dwarfs as a consequence of their low surface gravity. TWA22 A and B values are reported as red and green lines, respectively, with their associated uncertainties (dashed lines). If we assume a spectral type M6 \pm 1 for both components, the pseudo-equivalent widths of TWA22 AB appear intermediate between values found for young and field dwarfs.

and a young M5.5 dwarf from the Orion nebulae (age 1-2 Myr, S04). Together with the other age indicators, these intermediate surface gravity features confirm that TWA22 AB is likely to be a young binary system. However, their uncertainties remain large enough not to assign a precise age.

\subsection{Gravity and effective temperature from atmospheric models}

For a fine determination of the effective temperatures and surface gravities of TWA22 A and B, we compared our observed spectra with theoretical templates from the GAIA model v2.6.1 (Brott \& Hauschildt 2005). This library is updated from Allard et al. (2001). It benefits from improved molecular dissociation constants, additional dust species with opacities, spherical symmetry, and a mixing length parameter $2.0 \times H_{\mathrm{p}}$. The temperature ranges in the templates from 2000 to $10000 \mathrm{~K}$ and the gravity from -0.5 to 5.5 , but we limited our analysis to $2000 \mathrm{~K} \leq$ $T_{\text {eff }} \leq 4000 \mathrm{~K}$ and $3.5 \leq \log (g) \leq 5.5$. Theoretical spectra were 
convolved with a Gaussian to match the SINFONI spectral resolution and interpolated to the TWA22 AB wavelength grid. Least squares minimization was applied to find templates that fit the TWA22 A and B continuum avoiding zones polluted by remaining oscillations.

The TWA22 A least-square map in the $J$ band constrains the temperature between 2800 to $3100 \mathrm{~K}$ and is minimized for $\log (g)=4.5$ and $T_{\text {eff }}=2900 \mathrm{~K}$. In $H+K$ band, our minimization failed to reproduce the TWA22 A spectra faithfuly and makes us suspect the existence of a constant flux loss in the $H$ band during the spectral extraction process. To limit this systematic effect, the minimization was applied separately in $H$ and $K$ bands. In $H$-band, the effective temperature is minimized between $2600 \mathrm{~K}$ to $3000 \mathrm{~K}$ in the full space of surface gravities explored. The minimum is located at $2800 \mathrm{~K}$ and $\log (g)=4.5$. The $K$ band is reproduced by 2900 and $3000 \mathrm{~K}$ templates irrespective of gravity. Summing the three bands, we estimate an effective temperature $T_{\text {eff }}=2900_{-200}^{+200} \mathrm{~K}$ for TWA22 A. Conducting a similar analysis for the component TWA22 B, we derive an effective temperature $T_{\text {eff }}=2900_{-100}^{+200} \mathrm{~K}$. Using the Luhman et al. (2003) scale, these temperatures estimations respectively correspond to $\mathrm{M} 7_{-2}^{+1}$ and $\mathrm{M} 7_{-2}^{+0.5}$ spectral types for TWA22 A and B. This is also consistent with spectral types estimated in Sect. 4.3.2.

For a fine determination of the surface gravity from synthetic spectra, we computed the equivalent widths of K I lines in the $J$ band on each spectra of TWA22 A and B. We then compared the values to TWA22 A and B to restrain the acceptable gravity domain (see Fig. 11). We then estimated that the surface gravity is located between $\log (g)=4.0$ and 5.5 for TWA22 A and B.

\section{Discussion}

\subsection{Evolutionary models predictions}

The membership of TWA22 AB to TW Hydrae constrains the age of the system to 3-20 Myr (Barrado Y Navascués 2006; Scholz et al. 2007; de la Reza et al. 2006). Based on our astrometric observations combined with an accurate distance determination, we were able to derive the dynamical mass of this tight binary. From photometry and spectroscopy, we derived near-IR fluxes, luminosity, spectral type, effective temperatures, and the surface gravity of each component. Finally, spectroscopy tends to indicate that both components have intermediate surface gravity features in their spectra, supporting a young age for TWA22 AB. Assuming the TWA age for this system, we can now compare the measured total dynamical mass of the binary with the total mass predicted by evolutionary models of Baraffe et al. (1998; hereafter BCAH98). Model predictions are based on the $J H K$ photometry, the luminosity and the effective temperature of both components (see Fig. 12). At 8 Myr, BCAH98 models systematically underestimate the total mass by a factor of $\sim 2$. This factor varies from 3 to 1.3 between 3-5 Myr and $20 \mathrm{Myr}$. The mass is still strongly under-estimated using other evolutionary models of very low-mass stars (D'Antona \& Mazzitelli 1994, 1997). Alternatively, if we artificially change the system age to $30 \mathrm{Myr}$, the model predictions match our observations relatively well.

The apparent discrepancy between our observations and the model predictions at the age of TWA leads us to consider four explanations:

1. remaining uncertainties are present in our data reduction and interpretation related to the astrometry, photometry, and spectroscopy extraction process, the atmosphere model used or the assumption on the system itself;

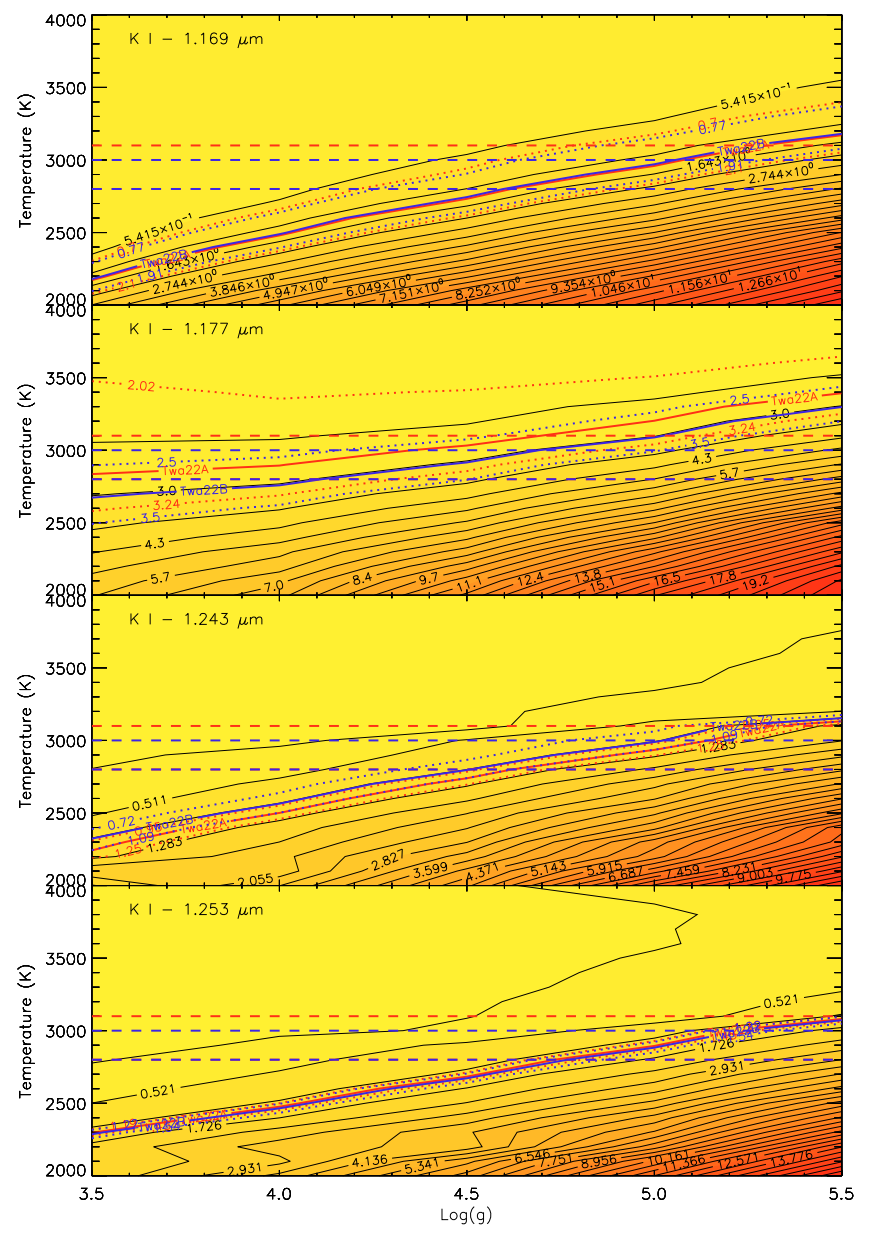

Fig. 11. Iso-contours plots of K I lines equivalent widths computed on each spectral template of the GAIA library v2.6.1 Red color indicates high values. The contours for TWA22 A (red) and B (blue) pseudoequivalents widths values are overplotted. The long-dashed lines represent limits on TWA22 A (red) and TWA22 B (blue) temperatures. Gravity is estimated inside these temperatures boxes.

2. the system has higher multiplicity than observed;

3. evolutionary model predictions are correct and the age estimate of TWA22 AB is currently incorrect. TWA22 AB would then be slightly older and aged of $30 \mathrm{Myr}$;

4. finally, the TWA22 AB age is 8 Myr and evolutionary models themselves do not correctly predict the physical properties of very low-mass stars at young ages.

Before drawing important conclusions on the validity of evolutionary models at young ages and very low masses, we consider below the first three explanations.

\subsection{Data reduction and interpretation uncertainties}

Systematics on the estimation of the relative position and near-IR fluxes of TWA22 A and B seems very unlikely. Our analysis relies on several imaging analysis techniques (aperture photometry, PSF fitting, deconvolution), already used and tested in various contexts. The tight binary TWA22 AB does not itself represent a difficult case. In addition, at each epoch, consistent results were found on several observing sequences obtained during the night.

Systematics in the spectroscopic observation and extraction seem more probable for determining effective temperature and surface gravity. Differential flux losses over the $J$ or $H+K$ 


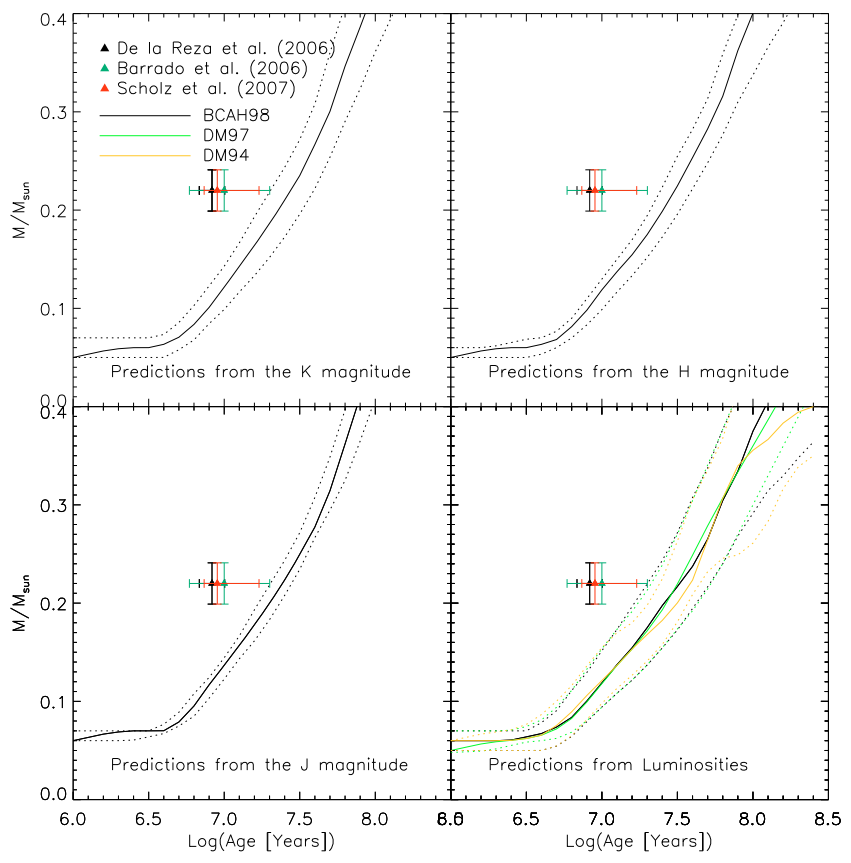

Fig. 12. Top-left: comparison of the binary direct mass measurement for different TW Hydrae age estimations (de la Reza et al. 2006; Barrado Y Navascués 2006; Scholz et al. 2007) with the predicted masses of the BCAH98 tracks derived from $M_{K}$. Errors on the photometry are propagated on predictions (dotted lines). Bottom-left: same as top-left but for predictions from $M_{H}$. Top-right: same as top-left but for predictions from $M_{J}$. Bottom-right: Same as top-left but for predictions from our estimated TWA22 A/B luminosities. In this plan, predictions from BCAH98, D'Antona \& Mazzitelli (1994), and D'Antona \& Mazzitelli (1997) models are nearly the same within our uncertainties.

spectral range may have occurred because of the limited size of the SINFONI field of view. The impact of this effect can be simulated by adding a linear slope in our spectral minimization over the different spectral bands. The results do not change our analysis significantly based on continuum fitting or spectral indexes. It does not affect the study of narrow lines and our surface gravity estimation at all. A nonlinear differential flux loss could be responsible for our failure to faithfully reproduce the TWA22 A and B spectra in $\mathrm{H}$-band using either empirical or synthetic libraries. Finally, the atmosphere models were also used in various conditions (metallicity, mixing length, different opacity tables) without drastically changing our results.

\subsection{Higher multiplicity hypothesis}

Considering that our data reduction and analysis are robust, we may wonder whether our basic assumptions concerning the system itself are correct. Actually, we cannot exclude from our observations that TWA22 AB is a multiple system of a higher order. One or even both components could be in fact unresolved binaries. In such a case, the derived effective temperatures, as well as the estimated spectral types, would not be strongly modified.

Dynamically speaking, the stability of the system would require the separation of the individual subcomponents to be significantly less than the size of the main orbit, typically by a factor 3-4 (Artymowicz \& Lubow 1994). To refine this estimate in the present case, we performed 3-body simulations using the symplectic code HJS (Beust 2003) dedicated to hierarchical systems. We assumed the fitted orbit and split one of the two components into 2 equal mass bodies, with a coplanar orbit with respect to the wide orbit and a given semi-major axis, and assumed initial zero eccentricity. We find that the system remains stable up to a separation of $\sim 0.4$ AU between the two subcomponents. This is a priori the most stable configuration, because a split into unequal masses would lead to less stability for the lighter component. We also checked that highly inclined relative configurations are physically unstable: they inevitably lead to a strong Kozai resonance characteristic for triple systems (Kozai 1962; Beust et al. 1997) that cause the eccentricity of the inner orbit to be pumped up to $\sim 1$, leading to a physical collision between the two individual components. Because of this, $0.4 \mathrm{AU}$ can be considered as the widest possible separation for hypothetical sub-components. This agree with Artymowicz \& Lubow (1994).

A separation of $0.4 \mathrm{AU}(\sim 22$ mas) remains below the PSF of the VLT/NACO images ( $\sim$ AU given the distance of TWA22). This would explain why the inner binary would not be resolved. However, we did not notice any PSF-lengthening in the images. With a separation of $0.4 \mathrm{AU}$, we could expect to see one. Does it suggest that the actual separation is significantly smaller?

An orbit with $0.4 \mathrm{AU}$ separation would correspond to an orbital period of $0.8 \mathrm{yr}$ and a radial velocity wobble of $\pm 7 \mathrm{~km} \mathrm{~s}^{-1}$ if we take the $27^{\circ}$ inclination into account with respect to the plane of the sky. Even though unlikely, this modulation could not have been detected during the monitoring (split up into two periods of 6 and 1 months). But if we assume a separation of $\sim 0.1 \mathrm{AU}$ to be compatible with the absence of PSF-lengthening, now the radial velocity wobble jumps to $\pm 14 \mathrm{~km} \mathrm{~s}^{-1}$ over a $0.1 \mathrm{yr}$ period. Such a variation was not detected in the radial velocity dataset (see Teixeira et al. 2009). Finally, no photocenter scatter is present around our two-body orbital solution. A motion of $\sim 5$ mas is expected along the orbital period for a separation of $\sim 0.1$ AU between the subcomponents.

Ultimately, we cannot definitely rule out the possibility that at least one of the two components of TWA22 is itself a binary, consisting of two nearly equal mass bodies. But combined dynamical and observational constraints show that the range of possible separations is fairly narrow, typically $0.1-0.2 \mathrm{AU}$. Also the system needs to be at least roughly coplanar.

\subsection{Age and membership of TWA22 AB}

Given the good agreement between observations and model predictions at $30 \mathrm{Myr}$, we can consider that the current age estimate of TWA22 AB is possibly incorrect. This age is currently inferred from the membership to TWA. Since the age of TWA is well established at $8 \mathrm{Myr}$ from various age diagnostics, a reliable explanation concerns the membership to TWA itself.

S03 identified TWA22 as a new member of TWA mainly from the observed Li absorption line at $6708 \AA$ and $\mathrm{H} \alpha$ emission line. The Li line is stronger $(E W=510 \mathrm{~m} \AA)$ than those of early-M dwarfs members of $\beta$ Pic, which led S03 to suggest an age $\leq 10$ Myr (see Fig. 8 of S03). They derived in addition a photometric distance of $22 \mathrm{pc}$ for TWA22, confirming the proximity of this young system. More recently, Mamajek (2005) has discussed the membership of TWA22 AB to TWA based on its kinematics properties and estimates a probability of $2 \%$ for TWA22 to be a member of TWA from an implemented convergence point technique (de Bruijne 1999). However, Song et al. (2006) mentioned that the strong $\mathrm{Li}$ line of TWA22 AB is observed only for young active $\mathrm{M}$ dwarfs in the direction of TWA with the exception of a very few M-type members of the $\beta$ Pictoris moving group (BPMG). Finally, Mentuch et al. (2008) obtained a 
new visible high-resolution spectrum of TWA22 AB. They confirm the strong equivalent line of the $6708 \AA$ lithium absorption $(E W=616 \pm 21 \mathrm{~m} \AA$, the strongest measured in their sample composed of young association members). They estimate $T_{\text {eff }}$ of $2990 \pm 13 \mathrm{~K}$ (compatible with the individual $T_{\text {eff }}$ derived in Part 4.4) and $\log (g)=4.2 \pm 0.05$ dex for the unresolved system. These new elements tend to confirm that TWA22 is a young system (age $\leq 30 \mathrm{Myr}$; see BCAH98 predictions).

To reconcile past and present results, we can consider the possibility that TWA22 AB is a member of the BPMG. With an $\mathrm{M} 6 \pm 1$ spectral type, TWA22 AB is probably close to the Lidepletion boundary (LDB) of TWA or $\beta$ Pic, which could possibly explain a significantly stronger $E W(\mathrm{Li})$ than those observed for early-M dwarfs of these two associations. We also notice that the $\lambda 6708 \AA$ line shows some variations between the S03 and M08 measurements. In addition, the observed $E W\left(\mathrm{H}_{\alpha}\right)$ of TWA22, used as a second indicator of youth, is compatible with those of GJ799 A and B, M4.5 members of $\beta$ Pic (Jayawardhana et al. 2006).

Finally, the projected position of TWA22 AB reveals that the system is isolated from other members of TWA. Its distance is more compatible with the mean distance of the BPMG members. Teixeira et al. (2009) have recently measured the proper motion, the trigonometric parallax, and the mean radial velocity of TWA22 AB. They determined for the first time the heliocentric space motion of TWA22 AB. From a detailed kinematic analysis they did not rule out TWA22 from TW Hydrae but demonstrated that it was a more probable member of the BPMG.

\section{Conclusions}

NACO resolved for the first time the young object TWA 22 as a tight binary with a projected separation of 1.76 AU. $80 \%$ of the binary orbit was covered during a 4-year observation program conducted with this instrument. We inferred a $220 \pm 21 M_{\text {Jup }}$ total mass for the system and obtained the individual magnitudes of each component in the near infrared. This places TWA22 A and $\mathrm{B}$ at the substellar boundary. We complete the characterization of the system components with medium resolution individual SINFONI spectra in the $J, H$, and $K$ bands. Our spectra were compared with the empirical library of young and field $M$ dwarfs. We derived an M6 \pm 1 spectral type from continuum fitting, spectral indexes and equivalent widths. Spectral templates were also used to estimate $T_{\text {eff }}=2900 \pm 200 \mathrm{~K}$ for TWA22 A and $T_{\text {eff }}=2900_{-100}^{+200} \mathrm{~K}$ for TWA22 B, and the surface gravity was constrained to $4.0<\log (g)<5.5 \mathrm{dex}$. These fundamental properties can be directly compared with commonly used evolutionary tracks provided that the age of the system is known accurately.

The age of TWA22 was still a matter of debate at the beginning of our study. TWA22 was reported as a member of the young association TW Hydrae, and alternatively as a possible member of the BPMG. At the age of TW Hydrae and BPMG, the new and precious benchmark brought by this system seems to point to an underestimation of the predicted mass from our photometry. However, the dynamical mass appears correctly estimated by the models if we consider a $30 \mathrm{Myr}$ old system. This led us to reconsider the membership of TWA22.

While the spectroscopy tends to confirm the youth of this system, a recent kinematic study rejected TWA22 as a member of the TW Hydrae and of the 30 Myr old Tucana-Horologium associations. It did not exclude the membership of TWA22 to the BPMG. Also, we cannot rule out the possibility that TWA22 is not associated with any of these moving groups.

Finally, we do not firmly exclude that the TWA22 AB component could in fact be unresolved binaries with coplanar inner orbits characterized by semi-major axis lower than $0.4 \mathrm{AU}$. The model predictions would match the measured dynamical mass of a triple or quadruple system. In this context, future monitoring of TWA22 AB with improved angular resolution could allow the resolution of the hypothetical inner binaries.

Acknowledgements. We thank the referee for an excellent and thorough review, which helped to improve our manuscript considerably. We thank the ESO Paranal staff for performing the service mode observations. We also acknowledge partial financial support from the Agence National de la Recherche and the Programmes Nationaux de Plantologie et de Physique Stellaire (PNP \& PNPS), in France. We are grateful to Andreas Seifahrt, Laird Close, Eric Nielsen, Catherine L. Slesnick, Nadya Gorlova, Katelyne N. Allers, and Nicolas Lodieu for providing their spectra. This work would have not been possible without the NIRSPEC and UKIRT libraries provided by Ian S. McLean, Michael C. Cushing, and John T. Rayner. We also would like to thank Peter H. Hauschildt, France Allard, and Isabelle Baraffe for their input on evolutionary models and synthetic spectral libraries. Finally, we thank Carlos Torres, Michael Sterzik, and Ben Zuckerman, who gave use precious insights into the discussion.

\section{References}

Allard, F., Hauschildt, P. H., Alexander, D. R., Tamanai, A., \& Schweitzer, A. 2001, ApJ, 556, 357

Allers, K. N., Jaffe, D. T., Luhman, K. L., et al. 2007, ApJ, 657, 511

Artymowicz, P., \& Lubow, S. H. 1994, ApJ, 421, 651

Baraffe, I., Chabrier, G., Allard, F., \& Hauschildt, P. H. 1998, A\&A, 337, 403

Barrado Y Navascués, D. 2006, A\&A, 459, 511

Beust, H. 2003, A\&A, 400, 1129

Beust, H., Corporon, P., Siess, L., Forestini, M., \& Lagrange, A.-M. 1997, A\&A, 320,478

Boccaletti, A., Chauvin, G., Baudoz, P., \& Beuzit, J.-L. 2008, A\&A, 482, 939

Boden, A. F., Sargent, A. I., Akeson, R. L., et al. 2005, ApJ, 635, 442

Bonnet, H., Ströbele, S., Biancat-Marchet, F., et al. 2003, in Adaptive Optical System Technologies II, ed. P. L. Wizinowich, \& D. Bonaccini, Proc. SPIE, 4839, 329

Brott, I., \& Hauschildt, P. H. 2005, in The Three-Dimensional Universe with Gaia, ed. C. Turon, K. S. O'Flaherty, \& M. A. C. Perryman, ESA SP, 576, 565

Burgasser, A. J. 2007, AJ, 134, 1330

Chabrier, G., Baraffe, I., Allard, F., \& Hauschildt, P. 2000, ApJ, 542, 464

Chauvin, G., Lagrange, A.-M., Dumas, C., et al. 2005, A\&A, 438, L25

Close, L. M., Lenzen, R., Guirado, J. C., et al. 2005, Nature, 433, 286

Close, L. M., Thatte, N., Nielsen, E. L., et al. 2007, ApJ, 665, 736

Cushing, M. C., Rayner, J. T., Davis, S. P., \& Vacca, W. D. 2003, ApJ, 582, 1066

Cushing, M. C., Rayner, J. T., \& Vacca, W. D. 2005, ApJ, 623, 1115

Cutri, R. M., Skrutskie, M. F., van Dyk, S., et al. 2003, 2MASS All Sky Catalog of point sources, The IRSA 2MASS All-Sky Point Source Catalog, NASA/IPAC Infrared Science Archive,

http://irsa.ipac.caltech.edu/applications/Gator/

D’Antona, F., \& Mazzitelli, I. 1994, ApJS, 90, 467

D’Antona, F., \& Mazzitelli, I. 1997, Mem. Soc. Astron. Ital., 68, 807

de Bruijne, J. H. J. 1999, MNRAS, 306, 381

de la Reza, R., Jilinski, E., \& Ortega, V. G. 2006, AJ, 131, 2609

Devillard, N. 1997, The Messenger, 87, 19

Diolaiti, E., Bendinelli, O., Bonaccini, D., et al. 2000, in Adaptive Optical Systems Technology, ed. P. L. Wizinowich, Proc. SPIE Vol., 4007, 879

Dumas, C., Terrile, R. J., Brown, R. H., Schneider, G., \& Smith, B. A. 2001, AJ, 121,1163

Eisenhauer, F., Abuter, R., Bickert, K., et al. 2003, in Instrument Design and Performance for Optical/Infrared Ground-based Telescopes, ed. M. Iye, \& A. F. M. Moorwood, Proc. SPIE, 4841, 1548

Golimowski, D. A., Leggett, S. K., Marley, M. S., et al. 2004, AJ, 127, 3516

Gorlova, N. I., Meyer, M. R., Rieke, G. H., \& Liebert, J. 2003, ApJ, 593, 1074 Hillenbrand, L. A., \& White, R. J. 2004, ApJ, 604, 741

Högbom, J. A. 1974, A\&AS, 15, 417

Jayawardhana, R., Coffey, J., Scholz, A., Brandeker, A., \& van Kerkwijk, M. H. 2006, ApJ, 648, 1206

Kastner, J. H., Zuckerman, B., Weintraub, D. A., \& Forveille, T. 1997, Science, 277, 67 
Kirkpatrick, J. D., Barman, T. S., Burgasser, A. J., et al. 2006, ApJ, 639, 1120

Kozai, Y. 1962, AJ, 67, 591

Leggett, S. K., Allard, F., Berriman, G., Dahn, C. C., \& Hauschildt, P. H. 1996, ApJS, 104, 117

Lenzen, R., Hofmann, R., Bizenberger, P., \& Tusche, A. 1998, in Infrared Astronomical Instrumentation, ed. M. F. Albert, Proc. SPIE, 3354, 606

Lodieu, N., Hambly, N. C., Jameson, R. F., \& Hodgkin, S. T. 2008, MNRAS, 383,1385

Lucas, P. W., Roche, P. F., Allard, F., \& Hauschildt, P. H. 2001, MNRAS, 326, 695

Luhman, K. L., Stauffer, J. R., Muench, A. A., et al. 2003, ApJ, 593, 1093

Mamajek, E. E. 2005, ApJ, 634, 1385

Mathieu, R. D., Baraffe, I., Simon, M., Stassun, K. G., \& White, R. 2007, in Protostars and Planets V, ed. B. Reipurth, D. Jewitt, \& K. Keil, 411

McCaughrean, M. J., \& Stauffer, J. R. 1994, AJ, 108, 1382

McGovern, M. R., Kirkpatrick, J. D., McLean, I. S., et al. 2004, ApJ, 600, 1020

McLean, I. S., McGovern, M. R., Burgasser, A. J., et al. 2003, ApJ, 596, 561

Mentuch, E., Brandeker, A., van Kerkwijk, M. H., Jayawardhana, R., \& Hauschildt, P. H. 2008, ApJ, 689, 1127
Modigliani, A., Hummel, W., Abuter, R., et al. 2007, ArXiv Astrophysics e-prints

Mohanty, S., Jayawardhana, R., Huélamo, N., \& Mamajek, E. 2007, ApJ, 657, 1064

Prato, L., Simon, M., Mazeh, T., Zucker, S., \& McLean, I. S. 2002, ApJ, 579, L99

Rousset, G., Lacombe, F., Puget, P., et al. 2000, in Adaptive Optical Systems Technology, ed. P. L. Wizinowich, Proc. SPIE, 4007, 72

Scholz, A., Coffey, J., Brandeker, A., \& Jayawardhana, R. 2007, ApJ, 662, 1254 Schwartz, U. J. 1978, A\&A, 65, 345

Sembach, K. R., \& Savage, B. D. 1992, ApJS, 83, 147

Slesnick, C. L., Hillenbrand, L. A., \& Carpenter, J. M. 2004, ApJ, 610, 1045

Song, I., Zuckerman, B., \& Bessell, M. S. 2003, ApJ, 599, 342

Song, I., Schneider, G., Zuckerman, B., et al. 2006, ApJ, 652, 724

Stassun, K. G., Mathieu, R. D., \& Valenti, J. A. 2007, ApJ, 664, 1154

Teixeira, R., Ducourant, C., Chauvin, G., et al. 2009, A\&A, 503, 281

Torres, C. A. O., Quast, G. R., Melo, C. H. F., \& Sterzik, M. F. 2008, ArXiv e-prints, 808

Veran, J.-P., \& Rigaut, F. J. 1998, in Adaptive Optical System Technologies, ed. D. Bonaccini, \& R. K. Tyson, Proc. SPIE, 3353, 426

Zuckerman, B., \& Song, I. 2004, ARA\&A, 42, 685 